\title{
Extended Einstein relations with a complex effective temperature in a one dimensional driven lattice gas
}

\author{
Kumiko Hayashi and Shin-ichi Sasa \\ Department of Pure and Applied Sciences, University of Tokyo, Komaba, Tokyo 153-8902, Japan
}

(Dated: November 10, 2018)

\begin{abstract}
We carried out numerical experiments on a one-dimensional driven lattice gas to elucidate the statistical properties of steady states far from equilibrium. By measuring the bulk density diffusion constant $D$, the conductivity $\sigma$, the intensity of density fluctuations $\chi$, we confirm that the Einstein relation $D \chi=\sigma T$, which is valid in the linear response regime about equilibrium, does not hold in such steady states. Here, $T$ is the environment temperature, and the Boltzmann constant is set to unity. Recalling that the Einstein relation provided the first step in the construction of linear response theory, we attempt to extend it to a generalized form valid in steady states far from equilibrium. In order to obtain new relations among measurable quantities, we define a complex effective temperature $\Theta-i \Phi$ from studying the static response of the system to a slowly varying potential in space. Replacing $T$ in the Einstein relation by the real part of the effective temperature $\Theta$, we numerically confirm that the relation $D \chi=\sigma \Theta$ holds in the nonequilibrium steady states far from equilibrium that we study. In addition to this extended form, we find the new relation $(L / 2 \pi) c \chi=\sigma \Phi$, where $c$ represents the propagation velocity of density fluctuations.
\end{abstract}

PACS numbers: 05.40.-a, 66.30.Dn, 02.50.Ey

\section{INTRODUCTION}

In 1905, Einstein derived the relation $D=\mu T$ between the diffusion constant $D$ for non-interacting Brownian particles suspended in an equilibrium fluid of temperature $T$ and mobility $\mu$ with respect to an external force 1]. (Here, the Boltzmann constant is set to unity.) This relation is now called the 'Einstein relation'. Through the development of linear response theory, the original Einstein relation was extended to the form

$$
D \chi=\sigma T,
$$

which is valid for interacting many-body systems [2]. Here, $D$ should be considered as the bulk density diffusion constant, $\chi$ is the intensity of density fluctuations, and $\sigma$ is the conductivity.

The Einstein relation is a universal relation in the linear response regime about equilibrium. It represents the first step in the construction of nonequilibrium statistical mechanics in the linear response regime. With this in mind, if the Einstein relation can be extended to some form valid for nonequilibrium steady states (NESS) far from equilibrium, realizing an understanding of this form will be an important step in the construction of statistical mechanical theory of NESS.

With this motivation, because it is simpler to study one-particle NESS systems than many-body systems, we previously analyzed a one-particle Langevin equation with a tilted periodic potential [3]. In that study, it was found that there exists an extended Einstein relation with an effective temperature that can be measured independently of the diffusion constant and the differential mobility. Then, it is natural to ask whether that result for a Brownian particle in NESS can be extended to many-body interacting systems. In the present paper, we address this question by studying a one-dimensional driven lattice gas (DLG) [4, 5, 6] as an example of a many-body interacting system.

\section{MODEL}

Let $\eta_{x}$ be an occupation variable defined on each site $x$ of a one-dimensional periodic lattice $x=1,2, \cdots, L$. The variable $\eta_{x}$ takes the value 1 if the $x$-th site is occupied by a particle and 0 if it is empty. In order to describe the model simply, we introduce the variable $\eta_{L+1}$, which is assumed to be equal to $\eta_{1}$. The array of occupation variables $\left(\eta_{1}, \cdots, \eta_{L}\right)$ is denoted by $\eta$ and called the "configuration".

The time evolution of $\eta$ is described by the following rule: At each time step, randomly choose a nearest neighbor pair $x$ and $x+1$, where $1 \leq x \leq L$, and exchange the values of $\eta_{x}$ and $\eta_{x+1}$ with probability $c(x ; \eta)$ given by $c(x ; \eta)=\alpha \mathrm{e}^{-\frac{1}{2} \beta Q\left(\eta \rightarrow \eta^{x}\right)}$, where $\eta^{x}$ is the configuration obtained from $\eta$ through this exchange, $\beta=1 / T$ is the inverse temperature of the environment, and $Q\left(\eta \rightarrow \eta^{x}\right)$ represents the heat absorbed from the heat bath as a result of change of configuration $\eta \rightarrow \eta^{x}$ in one Monte Carlo step. The constant $\alpha$ is determined so that $c(x ; \eta) \leq 1$. The particle number $N=\sum_{x=1}^{L} \eta_{x}$ is conserved throughout the time evolution, and the averaged density $\bar{\rho}=N / L$ is a parameter of the model.

In this paper, we study the DLG for which

$$
Q\left(\eta \rightarrow \eta^{\prime}\right)=H_{0}\left(\eta^{\prime}\right)-H_{0}(\eta)-E \sum_{x=1}^{L} j_{x}\left(\eta \rightarrow \eta^{\prime}\right),
$$

where $E$ is a constant driving force, $H_{0}(\eta)$ is the equilibrium Hamiltonian $H_{0}(\eta)=-\sum_{x=1}^{L} \eta_{x} \eta_{x+1}$, and $j_{x}(\eta \rightarrow$ $\left.\eta^{\prime}\right)$ is the net number of particles hopping from $x$ to $x+1$ for the configuration change $\eta \rightarrow \eta^{\prime}$ in one Monte Carlo 


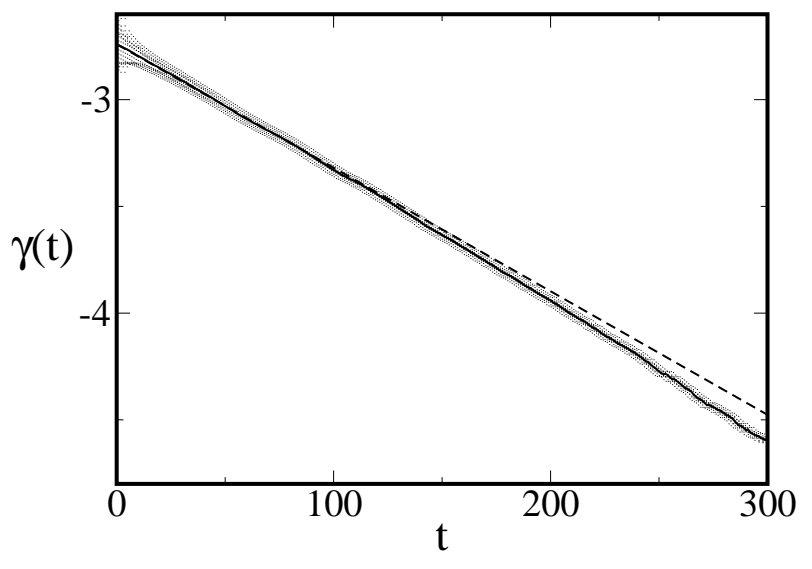

FIG. 1: $\gamma(t)$ is plotted with error bars in the case $\bar{\rho}=0.5$, $L=64$ and $\left|\hat{V}_{1}\right| / \sqrt{L}=0.1$ (See (10)). The slope of the fitted line (the dotted line) in the early stage $(t<150 \mathrm{mcs})$ corresponds to $D(2 \pi / L)^{2}$. The slope of $\gamma(t)$ in the late stage $(t>150 \mathrm{mcs})$ differs from $D(2 \pi / L)^{2}$.

step. In the argument below, \langle\rangle$_{E}^{\mathrm{s}}$ represents the statistical average in a NESS with driving force $E$.

Throughout this paper, we fix $\beta=0.5$, and the numerical parameter $\alpha$ introduced in the exchange probability $c(x ; \eta)$ is set to $\exp (-3 \beta)$. This value is chosen so that $c(x, \eta) \leq 1$ in the case $E \leq 5$. As a typical example of a NESS far from equilibrium, we study this model with $E=4$, and we consider the cases $\bar{\rho}=19 / 64$ and 0.5 , with $L=64,128$ and 256 . We remark that because we attempted to evaluate precisely measurable quantities introduced in the next section, it was hard to obtain data in the case $L>256$.

\section{EINSTEIN RELATION}

Because we have little knowledge regarding relations characterizing NESS of DLG, let us start by investigating the validity of the Einstein relation (1) by defining the quantities $\sigma, \chi$ and $D$ for this model. First, using the averaged current $\bar{J}_{E}$, which is defined by

$$
\bar{J}_{E}=\frac{1}{L}\left\langle\sum_{x=1}^{L} j_{x}\left(\eta \rightarrow \eta^{\prime}\right)\right\rangle_{E}^{\mathrm{s}},
$$

the conductivity $\sigma$ is expressed as

$$
\sigma=\frac{d \bar{J}_{E}}{d E}
$$

Second, the intensity of large scale density fluctuations is defined by

$$
\chi(L)=\left\langle\left|\hat{\eta}_{1}\right|^{2}\right\rangle_{E}^{\mathrm{s}},
$$

where

$$
\hat{\eta}_{n}(t)=\frac{1}{\sqrt{L}} \sum_{x=1}^{L} \eta_{x}(L t) \mathrm{e}^{i \frac{2 \pi n}{L} x}
$$

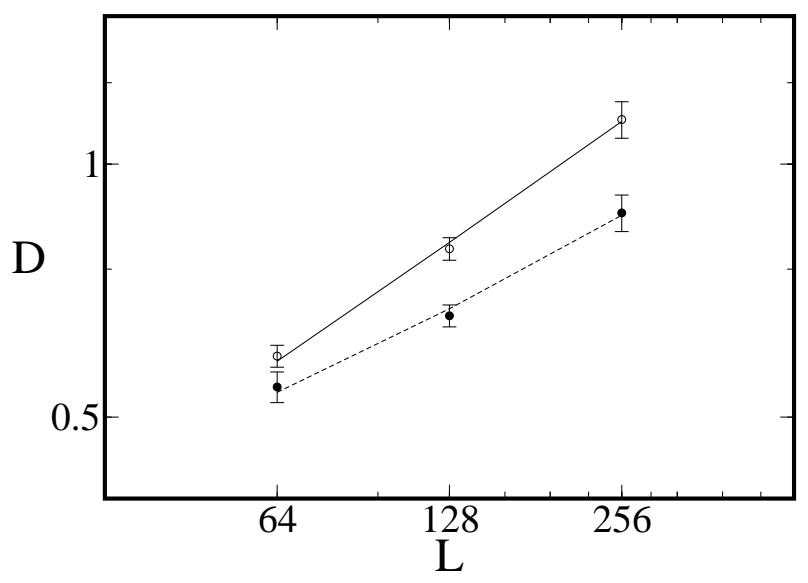

FIG. 2: $D$ as a function of $L$ for the case $\bar{\rho}=19 / 64$ (solid circles) and $\bar{\rho}=0.5$ (open circles). The dotted line and the solid line represent $0.042 \sqrt{L}+0.20$ and $0.068 \sqrt{L}+0.0413$, respectively.

Third, the density diffusion constant $D$ is defined as the coefficient of the diffusion term of the evolution equation describing the averaged behaviors of the density field. As an example of such behaviors, we consider the time evolution of the density field after a slowly varying weak potential $V_{x}$ is turned on at $t=0$ in the case that the system is in a steady state. Here $V_{x}$ is given by

$$
V_{x}=\frac{1}{\sqrt{L}} \hat{V}_{1} \mathrm{e}^{-i \frac{2 \pi}{L} x}+\text { c.c, }
$$

where c.c denotes the complex conjugate, and $\hat{V}_{1}$ is a complex number satisfying $\left|\hat{V}_{1}\right| / \sqrt{L} \ll 1$.

The averaged behavior in this case is represented approximately by the time evolution of a quantity $\left\langle\hat{\eta}_{1}(t)\right\rangle_{E}^{V}$, where \langle\rangle$_{E}^{V}$ denotes the statistical average under the condition that the equilibrium Hamiltonian becomes $H_{V}(\eta)=H_{0}(\eta)+\sum_{x=1}^{L} \eta_{x} V_{x}$ when $t>0$. We define the response function against $V_{x}$ as

$$
R(t)=-\lim _{\left|\hat{V}_{1}\right| \rightarrow 0} \frac{\left\langle\hat{\eta}_{1}(t)\right\rangle_{E}^{V}}{\hat{V}_{1}}
$$

In order to see the behavior of $R(t)$, in Fig. 1 we plot

$$
\gamma(t)=\log \left|-\frac{\left\langle\hat{\eta}_{1}(\infty)\right\rangle_{E}^{V}}{\hat{V}_{1}}+\frac{\left\langle\hat{\eta}_{1}(t)\right\rangle_{E}^{V}}{\hat{V}_{1}}\right|
$$

Note that we have checked that $\gamma(t)$ takes the same value for the cases $\left|\hat{V}_{1}\right| / \sqrt{L}=0.05,0.1,0.15$ and 0.2 . It implies that we can estimate the value of the right hand of (8) within a numerical accuracy. It is seen in Fig. 1 that $\gamma(t)$ has the linear slope in the early time regime. Therefore, using this linear slope, $D$ is estimated from the form

$$
|R(\infty)-R(t)|=\text { const. } \mathrm{e}^{-D\left(\frac{2 \pi}{L}\right)^{2} t},
$$




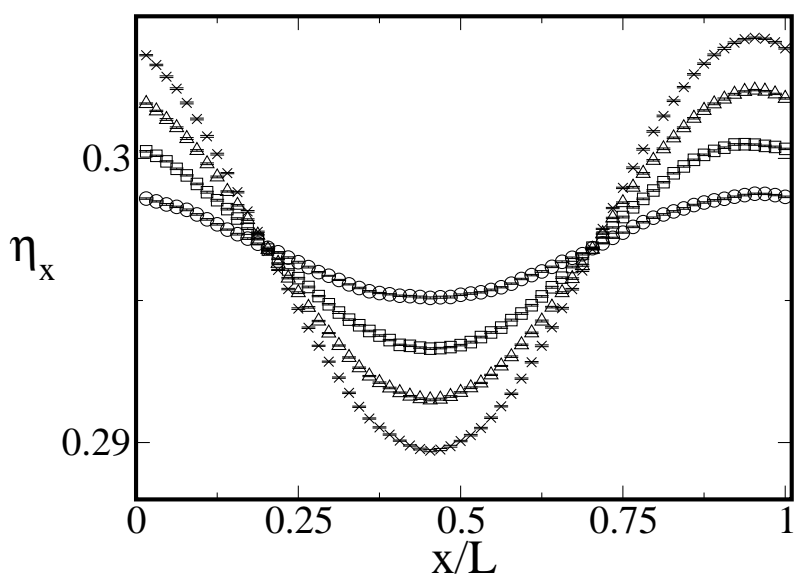

FIG. 3: The profiles of $\left\langle\eta_{x}\right\rangle_{E, V}^{\mathrm{s}}$ under the influence of $V_{x}=$ $\Delta \sin (2 \pi x / L)$ in the case $\bar{\rho}=19 / 64$ and $L=64 . \Delta=0.1$, $0.2,0.3$ and 0.4 correspond to the circle, square, triangle and plus, respectively.

in the early time regime. (We remark that we cannot deny a possibility that the linear slope shown in the early time regime might be caused by finite size effects. This is an unresolved problem.)

Using the above definitions of $\sigma, \chi$ and $D$, we confirm the validity of (11) numerically for the equilibrium case, $E=0$. With regard to other relations in the linear response theory, we can derive (1) from the Green-Kubo relation and the fluctuation response relation, which have been proved for DLG models [7]. Contrastingly, in NESS for our DLG, we find that the two sides of (11) differs greatly. Specifically, in the case $\bar{\rho}=19 / 64$, we have confirmed that $\sigma(L)$ and $\chi(L)$ converge to $\sigma_{*}=0.032$ and $\chi_{*}=0.26$ in the limit $L \rightarrow \infty$, while $D$ has the anomalous $L$ dependence $D(L) \propto \sqrt{L}$, as shown in Fig. 2 This anomalous behavior is essentially the same as that reported in Ref. [5]. Also, in the case $\bar{\rho}=0.5$, (10) is not valid because we have confirmed that $\sigma_{*}=0.037$ and $\chi_{*}=0.32$ in the limit $L \rightarrow \infty$, while $D(L) \propto \sqrt{L}$ (See Fig. (2).

Now we ask whether it is possible to obtain an extension of the Einstein relation valid for NESS of interacting many-body systems by defining an appropriate measurable effective temperature. This question arises naturally, because we have found that such an extended Einstein relation does hold for NESS of one-particle systems. In this relation, the temperature of the environment, $T$, is replaced by an effective temperature, which can be measured independently of the other quantities appearing in the Einstein relation [3]. By analogy to that case, we seek to define an effective temperature which when used in place of $T$ in (11) provides an extended Einstein relation that is valid for NESS in interacting many-body systems.

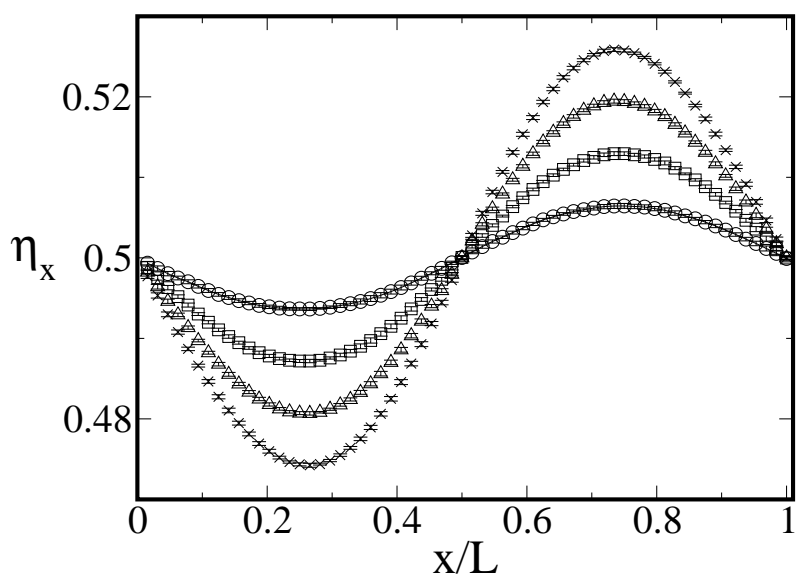

FIG. 4: The profiles of $\left\langle\eta_{x}\right\rangle_{E, V}^{\mathrm{s}}$ under the influence of $V_{x}=$ $\Delta \sin (2 \pi x / L)$ in the case $\bar{\rho}=0.5$ and $L=64 . \Delta=0.1,0.2$, 0.3 and 0.4 correspond to the circle, square, triangle and plus, respectively.

\section{EXTENDED EINSTEIN RELATIONS}

In a previous study, we showed that the effective temperature appearing in the Einstein relation valid for NESS of a Brownian particle can be measured by adding a slowly varying potential to the system [3]. In a similar way, we wish to determine an effective temperature by adding to the system a slowly varying potential given by (7). Due to the influence of this potential, we have $\left\langle\hat{\eta}_{1}\right\rangle_{E, V}^{\mathrm{s}} \neq 0$, where \langle\rangle$_{E, V}^{\mathrm{s}}$ denotes the steady state average in the system with driving force $E$ and potential $V_{x}$. Recalling that the relation

$$
\left\langle\hat{\eta}_{1}\right\rangle_{E=0, V}^{\mathrm{s}}=-\hat{V}_{1} \frac{\chi}{T}+o\left(\left|\hat{V}_{1}\right|\right),
$$

holds in the equilibrium case, we define the effective temperature $\hat{\theta}_{1}$ in NESS as

$$
-\lim _{\left|\hat{V}_{1}\right| \rightarrow 0} \frac{\left\langle\hat{\eta}_{1}\right\rangle_{E, V}^{\mathrm{s}}}{\hat{V}_{1}}=\frac{\chi(L)}{\hat{\theta}_{1}} .
$$

Note that in NESS, the left-hand side of (12) is a complex number in general. To see this concretely, we show that $\left\langle\eta_{x}\right\rangle_{E, V}^{\mathrm{s}}$ under the influence of the potential $V_{x}=\Delta \sin (2 \pi x / L)$ has a component of $\cos (2 \pi x / L)$ (see Fig. 3). Expressing explicitly that $\hat{\theta}_{1}$ is a complex number, we write

$$
\hat{\theta}_{1}=\Theta-i \Phi,
$$

where $\Theta$ and $\Phi$ are real numbers. In Fig. 5 . $\Theta$ and $\Phi$ are displayed as functions of $L$ in the case $\bar{\rho}=19 / 64$ and $\bar{\rho}=0.5$. It is seen that we have the anomalous $L$ dependences $\Theta(L) \propto \sqrt{L}$ and $\Phi(L) \propto L$. We remark that $\Phi=0$ in the case $\bar{\rho}=0.5$ (See Fig. (4).

Noting that from the graphs in Fig. 2]and Fig. [5 $D(L)$ and $\Theta(L)$ are both approximately proportional to $\sqrt{L}$, we 


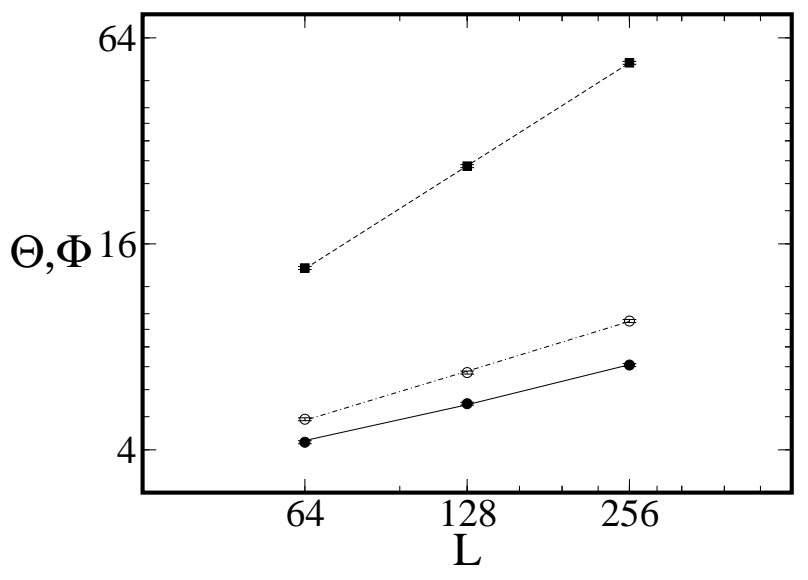

FIG. 5: $\Theta$ and $\Phi$ as functions of $L$ in the cases $\bar{\rho}=19 / 64$ (solid symbols) and $\bar{\rho}=0.5$ (open symbols). $\Theta(L)$ and $\Phi(L)$ correspond to the circle and square, respectively. The solid line, the dotted line and the dash-dotted line represents $0.36 \sqrt{L}+1.4$, $0.21 L$ and $0.58 \sqrt{L}+0.26$. Note that $T=2$.

investigate whether the Einstein relation can be extended to the NESS by simply replacing $T$ in (1) by $\Theta(L)$. In Fig. 6] we plot $D(L) \chi(L)$ as a function of $\sigma(L) \Theta(L)$ for the cases $\bar{\rho}=19 / 64$ and 0.5 , with $L=64,128$ and 256 . The graph clearly shows that the extended Einstein relation

$$
D(L) \chi(L)=\sigma(L) \Theta(L)
$$

holds within the precision of the numerical computations [8].

\section{FAILURE OF EXTENDED FLUCTUATION RESPONSE RELATION}

There are universal relations in the linear response regime about equilibrium, such as the Einstein relation, the fluctuation-response relation (FRR) and the GreenKubo relation. One may expect that other relations can be extended in the similar way as did for the Einstein relation (14) in NESS far from equilibrium. In this section, we investigate an extension of the FRR.

The FRR connects the time dependent response function and the time correlation function. In order to define the response function, we consider the situation that the perturbation (7) is applied to the system when $t=0$. The response function $R(t)$ in this situation is defined by (8). In the equilibrium case, we can prove the FRR

$$
R(t)=\frac{1}{T} C(t)
$$

with the time correlation function

$$
C(t)=\frac{1}{2}\left\langle\left|\hat{\eta}_{1}(t)-\hat{\eta}_{1}(0)\right|^{2}\right\rangle_{E}^{\mathrm{s}} .
$$

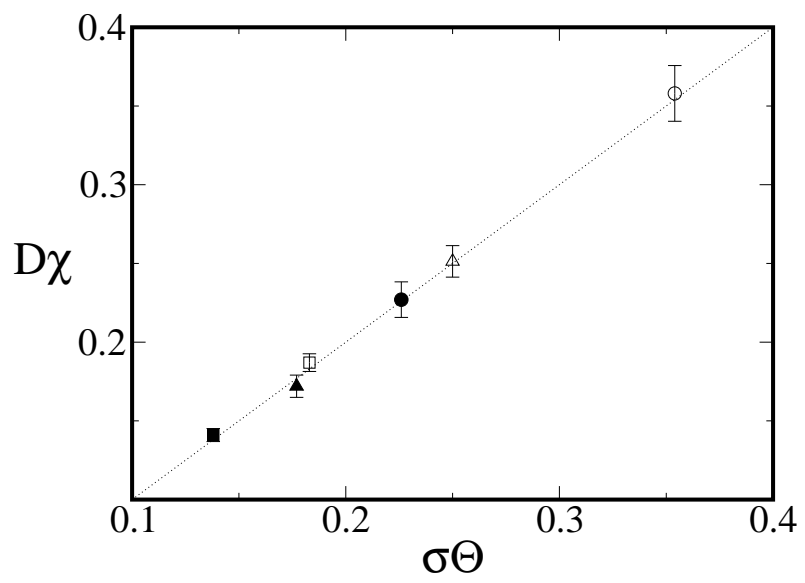

FIG. 6: $D \chi$ as a function of $\sigma \Theta$. The cases $\bar{\rho}=19 / 64$ and $\bar{\rho}=0.5$ correspond to the solid symbols and the open symbols, respectively. The cases $L=64,128$ and 256 correspond to the square, triangle, and circle, respectively. The straight line represents $D \chi=\sigma \Theta$.

Because the Einstein relation was extended with the replacement of $T$ by $\hat{\theta}_{1}$, we expect that (15) can be extended in the form

$$
R(t)=\frac{1}{\hat{\theta}_{1}} C(t)
$$

Specifically, we numerically investigate the validity of (17) in the case $\bar{\rho}=0.5$ and $L=64$. Because $\Phi=0$ in the case $\bar{\rho}=0.5$, (17) is rewritten as $R(t)=\Theta^{-1} C(t)$. In Fig. 7 we plot $R(t)$ as a function of $C(t)$ during an interval $0 \leq t \leq 800 \mathrm{mcs}$. It is seen that $R(t)$ deviates from $\Theta^{-1} C(t)$ substantially in an early time regime $(0 \leq t \leq 150 \mathrm{mcs})$ where the diffusion constant $D$ was defined (See (10)). This implies that (17) is not valid. Note that $R(\infty)=\hat{\theta}_{1}^{-1} C(\infty)$ holds by the very definition of $\hat{\theta}_{1}$ given by (12).

We remark that a generalized version of FRR of the nonlinear Langevin dynamics without the detailed balance condition was presented by several authors [17. However, in their formalism, a generalized force which defines a response function is chosen so that the simple form of FRR holds, and it seems that their response function cannot be measured directly in experiments. In contrast to the previous studies, we consider a relation between $C(t)$ and $R(t)$ which are measured directly.

\section{INTERPRETATION}

In sections $[\nabla$ and $\nabla$ we find that the Einstein relation (14) is extended with the diffusion constant $D$ which is measured from $R(t)$ in the early time regime and the effective temperature $\Theta$, while the extended FRR (17) is not valid unfortunately. In paragraphs below, we shall provide the phenomenological understanding of validity of (14) and invalidity of (17). 


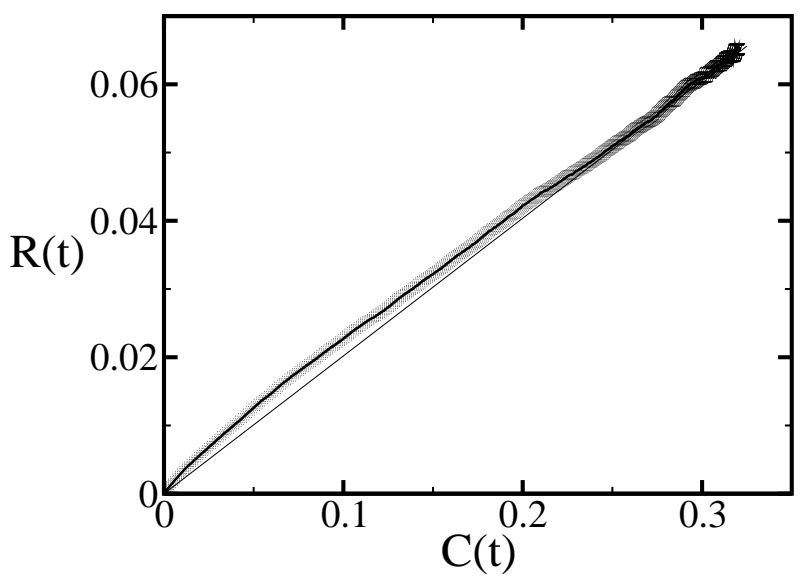

FIG. 7: $R(t)$ as a function of $C(t)$ in the case $\bar{\rho}=0.5$ and $L=64$ during an interval $0 \leq t \leq 800$ mcs. The slope of the thin line represents $\Theta^{-1}$.

The extended Einstein relation (14) can be explained from the following argument. Let $\bar{J}(\rho, E)$ be the averaged particle current in a system with density $\rho$ and driving force $E$. When the slowly varying potential (7) is applied to this system, first the current should become $\bar{J}\left(\left\langle\eta_{x}\right\rangle_{E, V}^{\mathrm{s}}, E-d V_{x} / d x\right)$, and as a result a diffusive current $D\left\langle\left(\eta_{x+1}-\eta_{x}\right)\right\rangle_{E, V}^{\mathrm{s}}$ should appear so as to make the total current homogeneous in $x$; that is, it is reasonable to assume the quantity

$$
\bar{J}\left(\left\langle\eta_{x}\right\rangle_{E, V}^{\mathrm{s}}, E-\frac{d V_{x}}{d x}\right)-D\left\langle\left(\eta_{x+1}-\eta_{x}\right)\right\rangle_{E, V}^{\mathrm{s}}
$$

is independent of $x$ in a steady state. This can be rewritten as

$c\left\langle\hat{\eta}_{1}\right\rangle_{E, V}^{\mathrm{s}}+i \sigma \frac{2 \pi}{L} \hat{V}_{1}+i \frac{2 \pi}{L} D\left\langle\hat{\eta}_{1}\right\rangle_{E, V}^{\mathrm{s}}=o\left(\frac{\left|\hat{V}_{1}\right|}{L}, \frac{\left\langle\hat{\eta}_{1}\right\rangle_{E, V}^{\mathrm{s}}}{L}\right)$,

where $c=\partial \bar{J}(\bar{\rho}, E) / \partial \bar{\rho}$ represents the propagation velocity of density fluctuations. From (12) and (19), we obtain

$$
\sigma \hat{\theta}_{1}=D \chi-i \frac{L}{2 \pi} \chi c .
$$

This leads to the extended Einstein relation (14).

Here, we note that a microscopic derivation of (18) is quite difficult. In particular, we have not been able to show that $D$ defined by (10) and $D$ in (18) are identical. In relation to this point, we note that a different value of $D$ is obtained if we consider the time regime $t \geq 150$ mcs in Fig. 11 Using this value of $D$, the relation (14) cannot be confirmed numerically. This result suggests that memory effects in a single mode description cannot be neglected in the late stage of the relaxation process after the potential is turned on. Because the memory effects arise from a nonlinear coupling between different

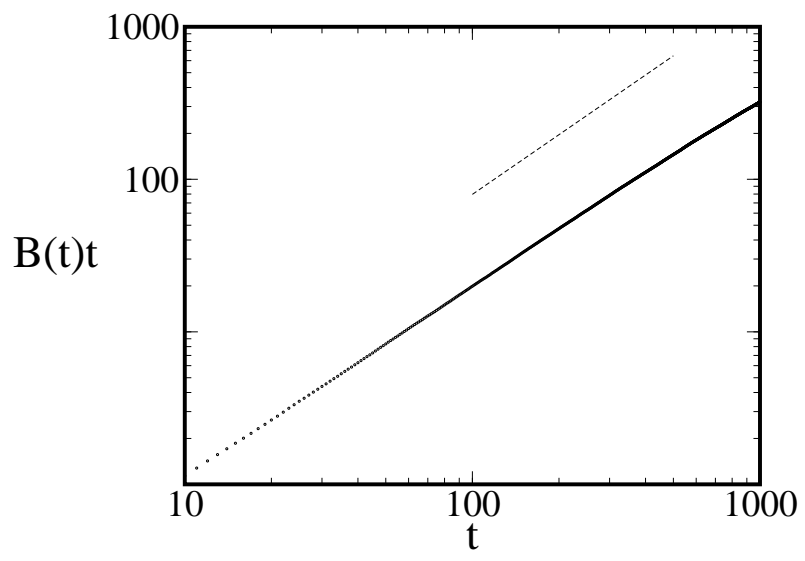

FIG. 8: $B(t) t$ as a function of $t$ in the case $\bar{\rho}=0.5$ and $L=$ 128. The guided line (the dashed line) represents $B(t) t \propto t^{4 / 3}$.

modes, the simple assumption (18) in NESS is far from the trivial.

The non-trivial nature of the extended Einstein relation (14) can be seen through the failure of the extended FRR (17). For example, because we cannot observe the exponential decaying regime of $C(\infty)-C(t)$ even in the early time regime unlike the behavior of $R(\infty)-R(t)$ (See Fig. 1), we cannot define the diffusion constant $D$ properly from the assumption that $\log |C(\infty)-C(t)|=-D\left(\frac{2 \pi}{L}\right)^{2} t+$ const., though in the equilibrium case, the same value of $D$ is obtained whether it is defined from $\log |C(\infty)-C(t)|$ or $\log |R(\infty)-R(t)|$ because of the FRR (15).

The non-exponential decaying behavior of $C(t)$ may be related to a long time tail of the time correlation function of current fluctuations. Indeed, using the continuity equation, $C(t)$ is expressed by

$C(t)=\left(\frac{2 \pi}{L}\right)^{2} \frac{1}{2}\left\langle\left|\sum_{k=1}^{L \tau} \sum_{x=1}^{L} j_{x}(\eta(k-1) \rightarrow \eta(k)) \mathrm{e}^{i \frac{2 \pi}{L} x}\right|^{2}\right\rangle_{E}^{\mathrm{s}}$

It is expected that the behavior of $C(t)$ in a shorter time regime than the diffusion time $D^{-1}(2 \pi / L)^{-2}$ has common features with that of

$$
B(t)=\frac{1}{2}\left\langle\left|\sum_{k=1}^{L t} \sum_{x=1}^{L}\left[j_{x}(\eta(k-1) \rightarrow \eta(k))-\bar{J}_{E}\right]\right|^{2}\right\rangle_{E}^{\mathrm{s}},
$$

which is displayed in Fig. 8 in the case $\bar{\rho}=0.5$ and $L=$ 128. The graph is consistent with the relation $B(t) t \propto$ $t^{4 / 3}$ which was derived from the theoretical analysis of fluctuating hydrodynamics [5].

We remark that these observations on $R(t)$ and $C(t)$ cannot be explained from the assumption that the time evolution of density field is described by a linear Langevin equation of $\hat{\eta}_{1}(t)$, because under this assumption both the relations (14) and (17) are derived though (17) was found not to valid. This implies that the dynamical aspect of 


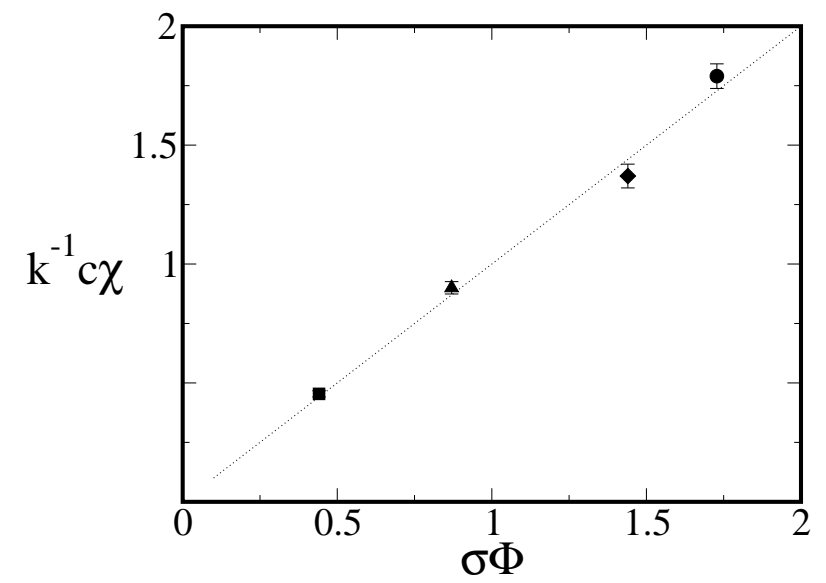

FIG. 9: $k^{-1} c \chi$ as a function of $\sigma \Phi$, where $k=2 \pi / L$, for the case $\bar{\rho}=19 / 64$. Note that for the case $\bar{\rho}=0.5, c=\Phi=0$. The cases $L=64,128,192$ and 256 correspond to the square, triangle, diamond and circle, respectively. The straight line represents $(L / 2 \pi) c \chi=\sigma \Phi$. The error bars represents the standard deviations of the statistical samples. In addition to the statistical error bars, there exists a fitting error which is less than 5 percent of the quantities.

the system is too complicated. It may be surprising that the extended Einstein relation holds with the appropriate choice of $D$.

Finally, from (20), we find a new relation

$$
\frac{L}{2 \pi} c(L) \chi(L)=\sigma(L) \Phi(L) .
$$

We investigated the validity of (23) in numerical experiments. We numerically measured $c$ from the oscillatory behavior of the time correlation function (16). In one example of this analysis, we considered all $t_{i}$ satisfying $C\left(t_{i}\right)-C(\infty)=0$, where $t_{i-1}<t_{i}$. Then, $c$ can be estimated from the relation

$$
c \frac{2 \pi}{L}\left(t_{i+1}-t_{i}\right)=\pi .
$$

We have confirmed that $c$ converges to $c_{*}=0.17$ in the case $\bar{\rho}=19 / 64$ in the limit $L \rightarrow \infty$. In Fig. 9 we plot $(L / 2 \pi) c(L) \chi(L)$ as a function of $\sigma(L) \Phi(L)$ for the cases $\bar{\rho}=19 / 64$, with $L=64,128$ and 256. (Note that $c=\Phi=0$ for the case $\bar{\rho}=0.5$.) This graph clearly shows that the extended Einstein relation (23) holds within the precision of the numerical computations.

\section{THERMODYNAMIC CONSIDERATION}

Here, we devote space to thermodynamic consideration related to our results. We first study the equilibrium case $E=0$ by considering a situation that a perturbation potential is applied to the equilibrium system. When we choose a slowly varying potential, we can regard $V_{x}$ and
$\left\langle\eta_{x}\right\rangle_{E=0, V}^{\mathrm{s}}$ as smooth quantities $V(x)$ and $\rho(x)$. By use of the chemical potential $\mu(\rho, T)$ of the equilibrium system (without the potential $V(x)$ ), a density profile under the influence of $V(x)$ is determined by a relation that

$$
\mu(\rho(x), T)+V(x)=\text { const. in } x,
$$

which is equivalent to

$$
\left(\frac{\partial \mu}{\partial \rho}\right)_{T} \frac{d \rho}{d x}+\frac{d V}{d x}=0
$$

Note that the fluctuation relation

$$
\left(\frac{\partial \mu}{\partial \rho}\right)_{T}^{-1}=\frac{\chi}{T}
$$

holds in the equilibrium state. As another relation in this situation, we have the total zero current condition

$$
-D \frac{d \rho}{d x}+\bar{J}\left(\rho,-\frac{d V}{d x}\right)=0 .
$$

By expanding the second term of (28) in $d V / d x$, we obtain

$$
D \frac{d \rho}{d x}+\sigma \frac{d V}{d x}=0
$$

A combination of (26), (27) and (29) leads to the Einstein relation (11). In the equilibrium case, we can show just the opposite that a combination of the Einstein relation (11) and the relation (29) derives the fluctuation relation (27) through the relation (25).

In connection with the above mentioned thermodynamic consideration, we introduce our previous study on a two-dimensional DLG [14]. First, we remark that an appropriate definition of the chemical potential for a NESS is not known, unless we use the local equilibrium assumption. Because in NESS far from equilibrium, the local equilibrium assumption is not valid, we attempted to define a chemical potential without this assumption. Focusing on a density response in a direction perpendicular to the driving force, we defined a nonequilibrium chemical potential by a similar relation with (25) and constructed an extended thermodynamic function from measurable quantities. As a non-trivial prediction, we showed numerically that the rate function of density fluctuation was determined by this extended thermodynamic function. A theoretical framework behind these observations has been uncovered [15]. We remark that the Einstein relation (11) was also observed in a direction perpendicular to the driving force [16] and that the argument in the previous paragraph can be applied to this case.

In the present paper, we have reported that the extended Einstein relation (14) and the new relation (23) hold in NESS, and a combination of both the relations and (19) that corresponds to the Fourier transform of (29) leads to the definition of the effective temperature (12). Because in the case $E=0$, the relation (11) is 


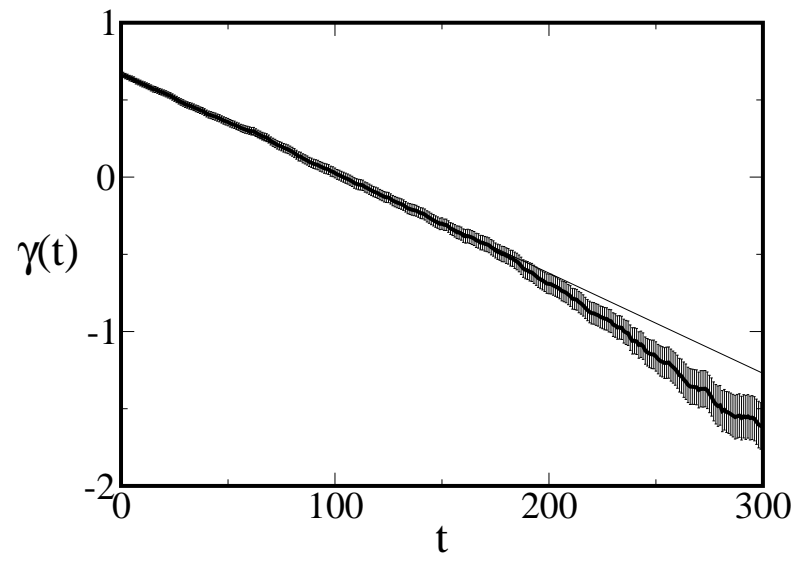

FIG. 10: In the case $H_{0}(\eta)=0$ (ASEP), $\gamma(t)$ is plotted with error bars in the case $\bar{\rho}=0.5, L=64$ and $\left|\hat{V}_{1}\right| / \sqrt{L}=0.1$ (see (10)). The slope of the fitted line (thin line) in the early stage $(t<150 \mathrm{mcs})$ corresponds to $D(2 \pi / L)^{2}$.

derived from (26) and (27), one might expect that there exists a nonequilibrium chemical potential even for the present NESS. For example, as a trial, one might propose a nonequilibrium chemical potential $\hat{\mu}_{1}\left(\hat{\rho}_{1}, \hat{\theta}_{1}\right)$ as

$$
\hat{\mu}_{1}\left(\hat{\rho}_{1}, \hat{\theta}_{1}\right)+\hat{V}_{1}=0 .
$$

However, (30) and (12) lead to

$$
\left(\frac{\hat{\mu}_{1}}{\hat{\rho}_{1}}\right)^{-1}=\frac{\chi}{\hat{\theta}_{1}}
$$

Because the relation (31) is hard to be interpreted as an extended form of the fluctuation relation (27), the definition (30) seems not to be useful. Taking into consideration that the relation (30) is just one trial, there might be a plausible definition of a chemical potential for NESS, but we have not found it yet. The difficulty that does not arise in the equilibrium case lies in the wavenumber dependence of $D$, as is expected from the system size dependence of $D$ shown in Fig. 2. This wavenumber dependence is not observed in the equilibrium case and prevents us from pushing ahead further thermodynamic consideration.

\section{DISCUSSION}

Equations (14) and (23) constitute the main results of this paper. In the following, we give five important remarks that are peripherally related to these main results.

First, we give an argument from which to understand a physical meaning of $\hat{\theta}_{1}$ as the effective temperature. Effective temperatures have been studied mainly in glassy systems [9, 10, 11, 12, 13. The simplest characterization of temperature may be provided by the equi-partition. The law of equi-partition is regarded as one example of an energy distribution law that can be expressed by the canonical distribution. Out of equilibrium, the equipartition law and the canonical distribution do not hold in general. Indeed, the steady distribution $p_{E}^{\mathrm{s}}(\eta)$ differs greatly from the canonical one. However, as demonstrated in Refs. [3, 10], by constructing measurement methods to extract behavior at some large time and/or length scales, the equi-partition law and the canonical distribution are recovered at these scales.

Hypothesizing a similar situation for the NESS we study, we assume a simple form for the modification of the steady state measure under the condition that the potential varying slowly in space is added to the system. The form we consider is

$$
p_{E, V}^{\mathrm{s}}(\eta)=\frac{1}{Z_{V}} p_{E}^{\mathrm{s}}(\eta) \mathrm{e}^{-\left(\hat{\eta}_{-1} \hat{\theta}_{1}^{-1} \hat{V}_{1}+\text { c.c. }\right)}
$$

for $\left|\hat{V}_{1}\right| \ll 1$, where $Z_{V}$ is a normalization constant. From (32), we can derive (12), which is the definition of $\hat{\theta}_{1}$. We believe that the simple form (32), which describes the response of the steady state measure to this slowly varying potential, is a good starting point to investigate NESS.

Second, we remark that our main result also holds for the case $H_{0}(\eta)=0$, which is an example of asymmetric simple exclusion process (ASEP). Specifically, we consider the case $\rho=0.5$ and $L=64 . \quad \chi$ and $\sigma$ are calculated theoretically as $\chi(L)=0.25$ and $\sigma(L)=0.043$. The diffusion constant $D(L)$ is measured numerically as $D(L)=0.68$ (see. Fig. 10). $\Theta$ is also measured as $\Theta(L)=3.9$ from (12). Thus the relation $D(L) \chi(L)=$ $\sigma(L) \Theta(L)$ seems valid. Study on the large scale behavior of the system might give new insights on ASEP.

Third, we remark a connection with fluctuating hydrodynamics. Note that the system in our numerical experiments may be too small for the dynamical behavior to be described within the framework of fluctuating hydrodynamics, while large enough so that we can check universal relations around equilibrium states. The fact that $D(L) \propto \sqrt{L}$ (See Fig 21) in such small systems seems to imply that bare parameters appearing in fluctuating hydrodynamics depend on a length scale $\ell$ at which hydrodynamic description is defined. (Note that the scaling relation $D(L) \propto \sqrt{L}$ can be derived within the framework of fluctuating hydrodynamics, irrespective of values of the bare parameters [5.) Thus, it is plausible to conjecture that we need to specify a scale $\ell$ explicitly so as to have a hydrodynamic description. We do not understand how the formal derivation in Ref. 17] corresponds to this observation.

Forth, we give a comment on diffusion of a marked particle. The diffusion is characterized by a tracer diffusion constant, which is not equal to the bulk diffusion constant, $D$, defined by (10). One may has a question whether the tracer diffusion constant can be involved in a universal relation for NESS. We do not find such a relation yet, but it may be an interesting future problem to seek it. We note that the tracer diffusion constant does 
not have the anomalous $L$ dependence in contrast to the case of $D$.

The final remark we wish to present regards the possibility of an experiment that can check the extended Einstein relations we have proposed. We conjecture that it may be best to study a system in which colloid particles, confined to one-dimension, are driven through a periodic array of optical traps [18]. By defining the effective temperature in the same way as did in (12), the validity of the extended Einstein relations will be checked.

The authors acknowledge H. Tasaki, T. Harada and M. Sano for stimulating discussions on NESS. This work was supported by a grant from the Ministry of Education, Science, Sports and Culture of Japan (No. 14654064).
[1] A. Eintein, Annalen der Physik 17 549, (1905); Investigations on the theory of Brownian movement, (Dover, 1956).

[2] R. Kubo, Rep. Prog. Theor. Phys. 29 255, (1966).

[3] K. Hayashi and S. Sasa, Phys. Rev. E 69, 066119, (2004).

[4] S. Katz, J. L. Lebowitz and H. Spohn, J. Stat. Phys. 34 497, (1984).

[5] H. van Beijeren, R. Kutner, and H. Spohn, Phy. Rev. Lett. 54 2026, (1985).

[6] H. Spohn, Large Scale Dynamics of Interacting Particles , (Springer-Verlag, 1991).

[7] K. Hayashi and S. Sasa, in preparation

[8] There is a puzzle that the extended Einstein relation seems to connect this $D$ with $\sigma$, because $D$ is evaluated form the short time behavior of the system, while $\sigma$ is measured as the long time average in the steady state.

[9] L.F. Cugliandolo, J. Kurchan and L. Peliti, Phys. Rev. E 55, 3898, (1997).
[10] L. Berthier and J-L Barrat, Phys. Rev. Lett., 89, 095702, (2002).

[11] A. B. Kolton, R. Exartier, L. F. Cugliandolo, D. Dominguez and N. Gronbech-Jensen, Phys. Rev. Lett., 89, 227001, (2002).

[12] I. K. Ono et al, Phys. Rev. Lett., 89, 095703, (2002).

[13] T. Harada, cond-mat/0310547 Europhysics Letters, in press.

[14] K. Hayashi and S. Sasa, Phy. Rev. E 68, 035104(R) (2003).

[15] S. Sasa and H. Tasaki, cond-mat/0411052

[16] K. Hayashi, in preparation.

[17] G. L. Eyink, J. L. Lebowitz, and H. Spohn, J. Stat. Phys. 83 385, (1996) and references therein..

[18] P. T. Korda, M. B. Taylor, and D. G. Grier, Phys. Rev. Lett. 89 128301, (2002). 